\title{
Flow Starting Point and Voiding Mechanisms Measured by Simultaneous Registrations of Intravesical, Intra-abdominal, and Intraurethral Pressures in Awake Rats
}

\author{
Long-Hu Jin ${ }^{*}$, Chang-Shin Park ${ }^{1, *}$, Dana Kim, Bo-Hwa Choi ${ }^{1}$, Soo-Hwan Park ${ }^{2}$, Sang-Min Yoon ${ }^{2}$, Tack Lee ${ }^{2}$ \\ Department of Urology, Shanghai Tenth People's Hospital, Tongji University School of Medicine, Shanghai, China; \\ Departments of ${ }^{1}$ Pharmacology and ${ }^{2}$ Urology, Inha University School of Medicine, Incheon, Korea
}

\begin{abstract}
Purpose: The aim of this study was to apply a new surgical procedure that allows for the successful monitoring of intraurethral pressure (IUP) changes in the cystometry of awake Sprague-Dawley rats.

Methods: Twenty-six female Sprague-Dawley rats were grouped according to the catheterization method (bladder only; bladder and urethra; or bladder, urethra, and abdomen). Using an arbitrarily determined initial point of the first phase among four rat micturition phases on the simultaneous curves as a reference point, we compared the time differences to the points on an intravesical pressure (IVP) and those on IUP or a detrusor pressure (DP) curve from intra-abdominal pressure (IAP).

Results: In awake rat, the start of urethral flow on IUP curve corresponded to the initial point of the second phase, which is same to the results on the anesthetized rat. However, certain results, such as micturition pressure (MP) and intraluminal pressure high-frequency oscillations (IPHFOs), differed between awake and anesthetized rats. Most MP values were checked after the end of urethral flow on the IUP curve, which is due to the peculiar methodology such as transvesical catheterization. Urethral flow was not completely interrupted during the IPHFOs, which suggests the presence of urethral wall tension against the flow during voiding. After removal of the superimposed effects of IAP from IVP, the DP curve clearly showed a peculiar shape, highlighting the possibility of using IAP in place of IUP to detect the flow starting point on the IVP curve.

Conclusions: Awake rat cystometry results have been interpreted based on those in anesthetized rats. However, our awake cystometry data were substantially different in terms of voiding time compared to those of anesthetized rats. This discovery warrants careful interpretation of the voiding parameters in awake rat cystometry.
\end{abstract}

Keywords: Urodynamics; Urinary bladder; Urethra; Rats

\section{INTRODUCTION}

Micturition results from complex interactions between the bladder and urethra [1,2], yet the alterations that affect these interactions and result in voiding dysfunction in various diseases have not yet been fully elucidated. In rat cystometry, understanding these reciprocal actions that result in bladder emptying requires careful interpretation of the unpredictable intravesical pressure (IVP) curve based on simultaneous urethral activity [3], which is primarily caused by intra-abdominal pressure (IAP) changes during the awake condition $[4,5]$. In anes- thetized rats, the dynamic nature of voiding has been well proven and documented based on observations of the simultaneous activities of the bladder and urethra [6-8]. However, controversy persists concerning interpretations of the IVP curve of awake animals during the voiding phase due to unpredictable IAP changes and the lack of a reliable method for measuring urethral activity in the awake condition [9].

On transvesical cystometry in anesthetized rats, the IVP curve shape during micturition can be divided into four distinct phases $[6,7,10]$. It has been well proven that urinary flow starts at the initial point of the second phase, followed by intraluminal
Corresponding author: Tack Lee

Department of Urology, Inha University Hospital, Inha University School of Medicine, 27 Inhang-ro, Jung-gu, Incheon 400-711, Korea

Tel: +82-32-890-3448 / Fax: +82-32-890-3097 / E-mail: lee.tack33@gmail.com "Long-Hu Jin and Chang-Shin Park contributed equally to this study as co-first authors.

Submitted: May 14, 2014 / Accepted after revision: June 11, 2014
This is an Open Access article distributed under the terms of the Creative Commons Attribution Non-Commercial License (http://creativecommons.org/licenses/by-nc/3.0/) which permits unrestricted non-commercial use, distribution, and reproduction in any medium, provided the original work is properly cited.

Copyright @ 2014 Korean Continence Society

www.einj.org 
pressure high-frequency oscillations (IPHFOs), and that the conventional concept of micturition pressure (MP) exists between the third and fourth phases [4-7]. However, this has never been described in awake rats due to the lack of an adequate method for assessing intraurethral pressure (IUP) in this condition. We recently developed a new method to check IUP directly from the distal urethra during the awake condition that captures the momentary start or finish of urethral emptying and enables comparison of the time sequences of some voiding events on simultaneous curves measuring different parameters.

Urethral manipulation to check IUP in conscious animals may change their natural voiding behavior because of the painsensitive characteristics of the urethra or the possibility of mechanical obstruction by the catheter tip within the urethra [11]. Thus, it would be desirable to have an alternative less invasive physiological measurement technique that accurately represents urethral activity. Such an alternative method would require confirmation that the IVP curve shape correlates with IUP parameters identified through this study. During micturition, abdominal straining is believed to be a normal physiological action that increases IAP and may eclipse the traces of detrusor pressure (DP) during voiding events [9,12-14]. Thus, we studied whether we can detect the flow starting point (FSP) using only the shape of the DP curve after removing the effect of IAP from IVP.

The aims of this study were to test the hypotheses that the FSP in awake rat cystometry occurs at the beginning of the second phase as in anesthetized rats and that MP occurs during the urethral flow observed by IUP. We also determined whether IAP can be used instead of IUP to detect FSP in awake female rats using a model in which the pressures were measured simultaneously in the bladder and distal urethra with or without perivesical space. Additional supportive information was obtained on IPHFOs during simultaneous recordings of IVP and IUP.

\section{MATERIALS AND METHODS}

\section{Experimental Animals}

Twenty-six female Sprague-Dawley rats weighing 230-260 g (Orient Bio Inc., Seongnam, Korea) were used in this study. The rats were randomly distributed into three groups. In group I $(\mathrm{n}=10)$, cystometry was performed using two catheters: one inserted into the bladder and one inserted into the urethra. In six of the 10 animals, successful IUP recordings were made (two rats died and two recordings were faulty) (Fig. 1A). In group II $(n=10)$, cystometry was performed using a balloon catheter to check the IAP in addition to the two catheters described above for group I (Fig. 1B). Successful recordings were made in six of 10 animals (three rats died and one recording was faulty). Group III $(n=6)$, in which only an IVP catheter was used, was used as a control group to investigate the effects of using additional IUP or IAP catheters (Fig. 1C). The rats were handled in standard laboratory conditions with a 12:12-hour light:dark cycle and free access to food pellets and tap water. All procedures
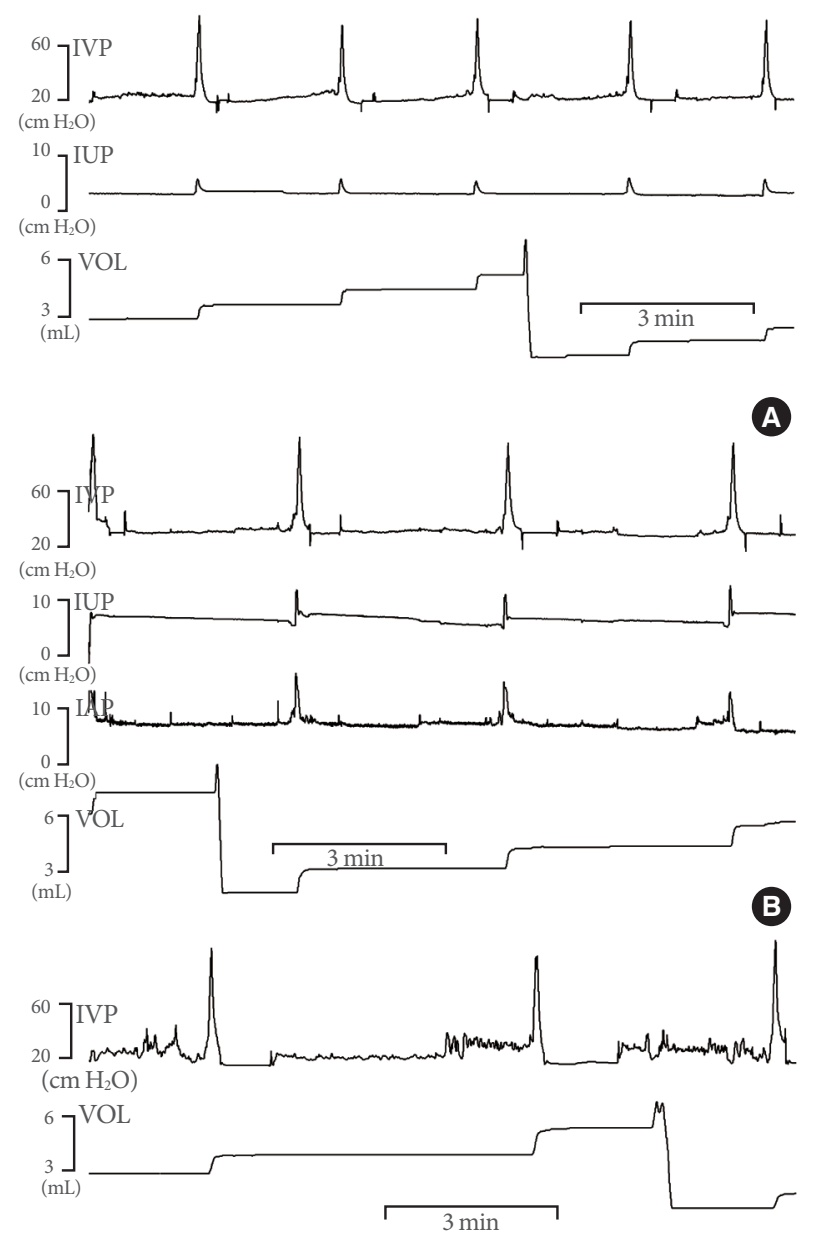

○

Fig. 1. Representative cystometric recordings in awake rats in which: two catheters were inserted into the bladder and urethra (group I, A); three catheters inserted into the bladder, perivesical area, and urethra (group II, B); or one catheter was inserted into the bladder (group III, C). Note that intraurethral pressure (IUP) decreased slightly before the start of urethral flow and showed the humps of IUP change only during urethral flow. Panel B shows the relationships between intravesical pressure (IVP), intra-abdominal pressure (IAP), and IUP and the flow of urine. Recorded as significant increases in IAP, all rats exhibited abdominal strain during every void. VOL, volume. 
for animal handling and treatment were performed in accordance with the Guide for the Care and Use of Laboratory Animals of the National Institutes of Health and were approved by the Ethics Committee of Inha University Hospital.

\section{Surgical Procedures}

The rats were anesthetized with ketamine $(75 \mathrm{mg} / \mathrm{kg}$ intraperitoneally; Yuhan Co., Seoul, Korea) and xylazine (15 mg/kg intraperitoneally; Rompun, Bayer Korea Co., Seoul, Korea). After surgery, the animals were caged individually and maintained in the same manner.

\section{Intravesical and Intra-abdominal Catheter Implantation}

The preparations and recording methods used are described in our previous papers [9,12-15]. Briefly, a polyethylene catheter (PE-50, Becton Dickinson, Parsipanny, NJ, USA) with a cuff was implanted in the bladder through an abdominal midline incision into the dome and held in place by a purse-string suture. To record IAP, a 0.05-mL latex balloon (Daewoo Medical,
Incheon, Korea), which was filled with distilled water and placed on the cuff of the PE-50 polyethylene catheter's tip, was inserted into the abdomen and placed around the IVP recording catheter using a silk tie.

\section{Intraurethral Catheter Implantation}

To record IUP, a PE-50 polyethylene catheter was elongated to about 1.5 times its original length at the tip of the inserting side and inserted into the urethral orifice according to the following method. Through the incised abdominal space, a 24-gauge angioneedle was inserted from the abdominal cavity to the urethral lumen around the urethral orifice. The needle stylet was removed and reinserted into the needle sheath in the opposite direction. After the sheath was removed, we slid the catheter over the remaining needle from the direction of the inside of the abdomen to the urethra. After the stylet was removed, the catheter tip was flared with a flame to create the tip cuff and secure it inside the urethral lumen (Fig. 2A). The proximal end of the catheter was tunneled subcutaneously and anchored to the
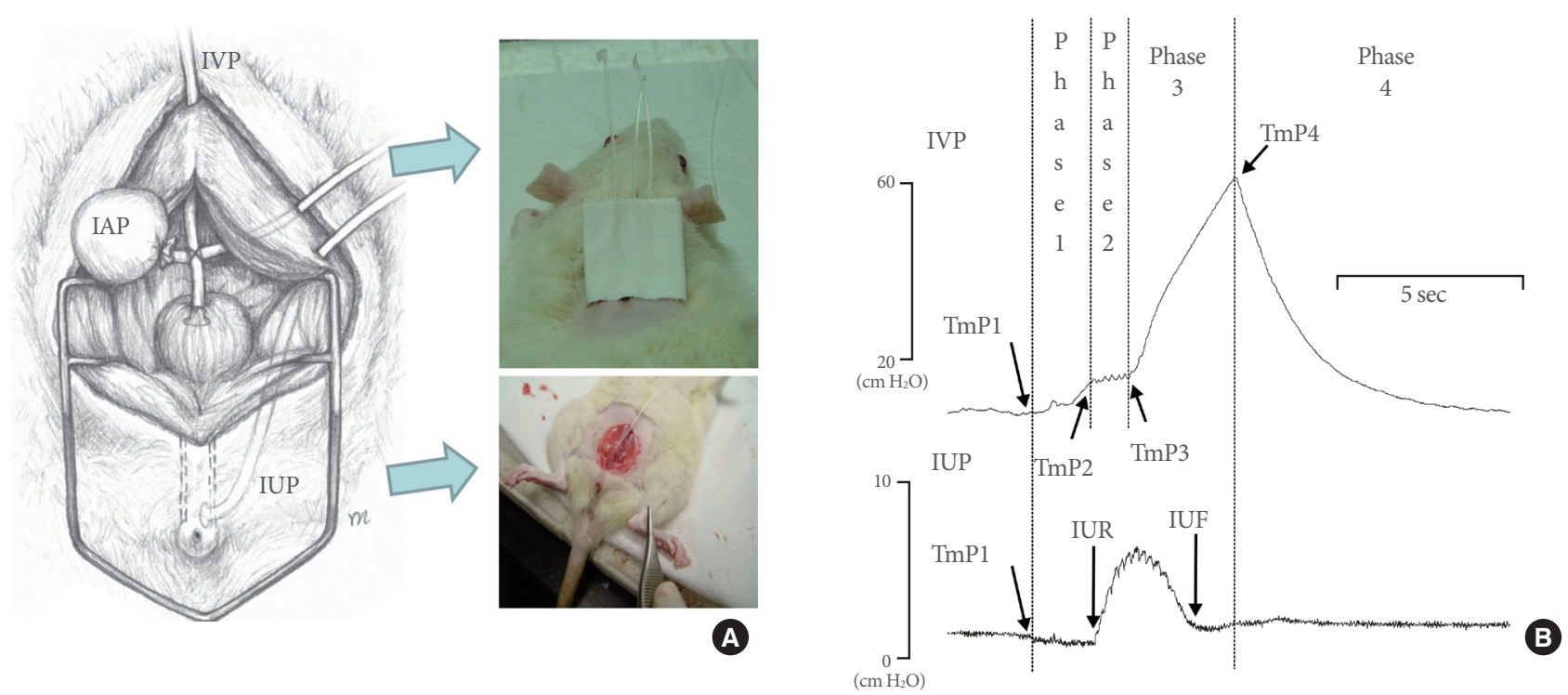

Fig. 2. Schematic diagram showing the positions of the intra-abdominal and intravesical catheters in the opened abdomen of a rat (A) and representative simultaneous pressure recordings during the voiding phase in awake rats with two catheters inserted into the bladder and urethra (B). The proximal ends of the catheters were tunneled subcutaneously and anchored to the skin of the back using a silk ligature. Through the abdominal incision, the urethral catheter was inserted from the abdominal cavity to the urethral lumen around the urethral orifice (A). The phases and time points on simultaneous curves of intravesical pressure (IVP) and intraurethral pressure (IUP) are shown (B). An initial point was arbitrarily determined just before the increase in IVP (TmP1), and the small top or end of this increase (phase 1) was defined as TmP2. This was followed by intraluminal pressure high-frequency oscillations showing pulsatile fluctuation on the IVP curve (phase 2). After that, the curve increased again (phase 3), and the initial point of this increase was defined as $\mathrm{TmP}$. The top of this increase was defined as $\mathrm{TmP} 4$, and there was a rapid pressure decrease to the low level seen before micturition contraction (phase 4). IAP, intra-abdominal pressure; TmP, time point; IUR, IUP rise; IUF, intraurethral pressure fall. 
skin of the rat's back using a silk ligature. The free end of the catheter was then sealed.

\section{Cystometric Investigations}

Cystometry was performed without anesthesia 3 days after catheter implantation. The conscious rats were placed in metabolic cages without restraint and the bladder catheter was connected via a T-tube to a pressure transducer (Research Grade Blood Pressure Transducer; Harvard Apparatus, Holliston, MA, USA) and a microinjection pump (PHD 22/2000 pump, Harvard Apparatus). Micturition volumes (MVs) were recorded with a fluid collector connected to a force displacement transducer (Research Grade Isometric Transducer, Harvard Apparatus). Room-temperature saline was continuously infused into the bladder at a rate of $20 \mathrm{~mL} / \mathrm{hr}$. Pressure and MVs were recorded continuously using Acqknowledge 3.8.1 software and an MP150 data acquisition system (BIOPAC Systems, Goleta, CA, USA) at a sampling rate of $100 \mathrm{~Hz}$. The average values from three reproducible micturition cycles of each rat from each group were used for urodynamic evaluation. IAP was defined as the recorded balloon pressure corrected by subtracting the lowest balloon pressure in each voiding cycle for zeroing. The DP was defined as the IVP minus the IAP.

The following urodynamic parameters were investigated: BP (the lowest bladder pressure during filling), FSP (the bladder pressure at the time of the start of flow of urine from the urethra) [9], threshold pressure (TP; bladder pressure immediately before micturition), MP (maximum bladder pressure related to micturition), bladder capacity (BC; residual volume $[\mathrm{RV}]$ at the most recent micturition plus the volume of saline infused at micturition), MV (volume of expelled urine), RV (BC minus $\mathrm{MV}$ ), and micturition interval.

\section{Time Points to Determine the Sequence of Voiding Events on Simultaneous IVP and IUP Recordings}

Fig. $2 \mathrm{~B}$ shows the details underlying the timing of various voiding events in simultaneous IVP, IUP, and volume curves. Time points (TmPs) on IVP were defined as follows. Among the four phases of rat micturition $[6,10]$, TmP1 was arbitrarily defined to occur just before the increase in the IVP (phase 1), and the small top or end of this increase was defined as TmP2. This was followed by IPHFOs showing pulsatile fluctuation of the IVP curve (phase 2) [4]. After that, the curve increased again (phase 3 ) $[6,7]$, and the initial point of this increase was defined as TmP3. The top of this increase was defined as TmP4, and there was a rapid pressure decline to the low level seen prior to micturition contraction (phase 4). This top of the increase was considered conventional MP. The TmP of the change in IUP was defined as the IUP rise (IUR) or IUP fall (IUF) (Fig. 2B), while the TmP of the rise on the volume curve was defined as the volume rise (VR) (Fig. 3A).
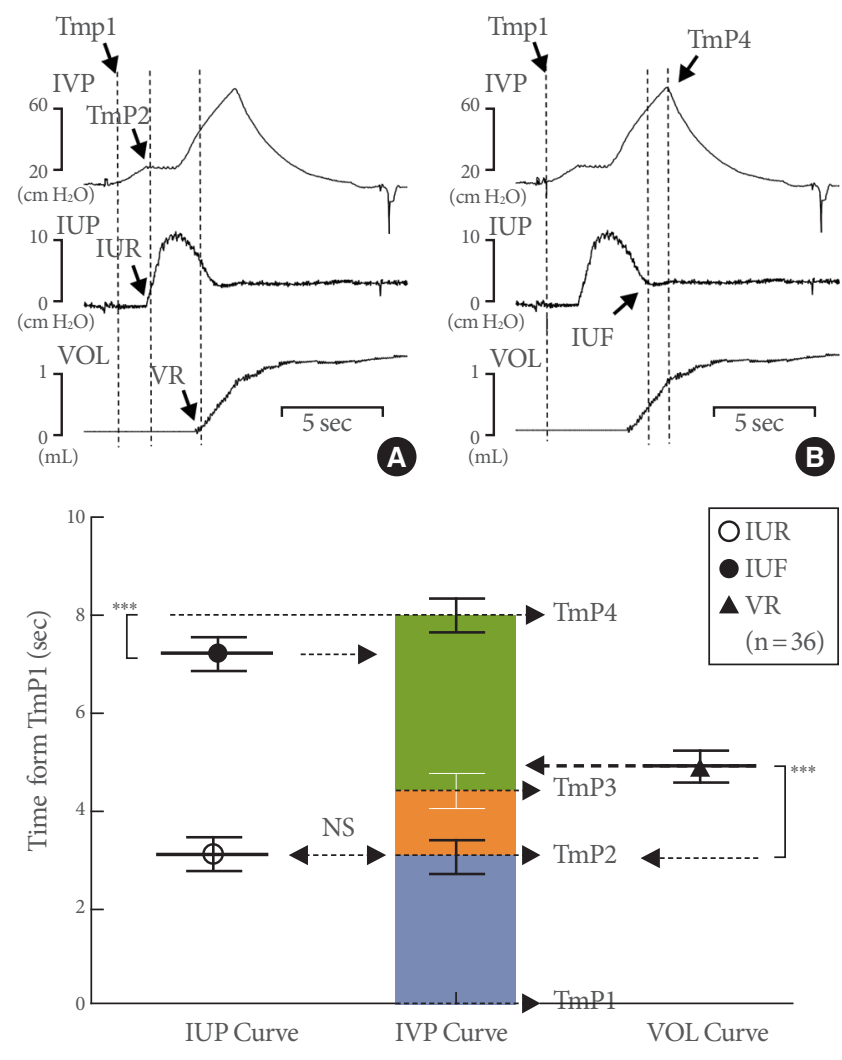

C

Fig. 3. Time differences from time point $1(\mathrm{TmP} 1)$ to various events on the simultaneous curves of intravesical pressure (IVP), intraurethral pressure (IUP), and volume (VOL). (A) Representative tracings showing the time to TmP2, IUP rise (IUR), and VOL rise (VR). The IUR on the IUP curve corresponds to TmP2 on the IVP curve, and the VR on the VOL curve was delayed compared to the time to TmP2 on the IVP curve. (B) Representative tracings showing the time to TmP4 and IUP fall (IUF). (C) Comparison of data from the curves of IUP, IVP, and VOL (mean \pm standard error). The time to IUF was shorter than the time to TmP4, suggesting that the voiding event known as micturition pressure occurs just after the end of urethral flow and may not correspond to real pressures during voiding. NS, not significant. ${ }^{* * *} \mathrm{P}<0.001$ (compared to the data from IVP curve). 

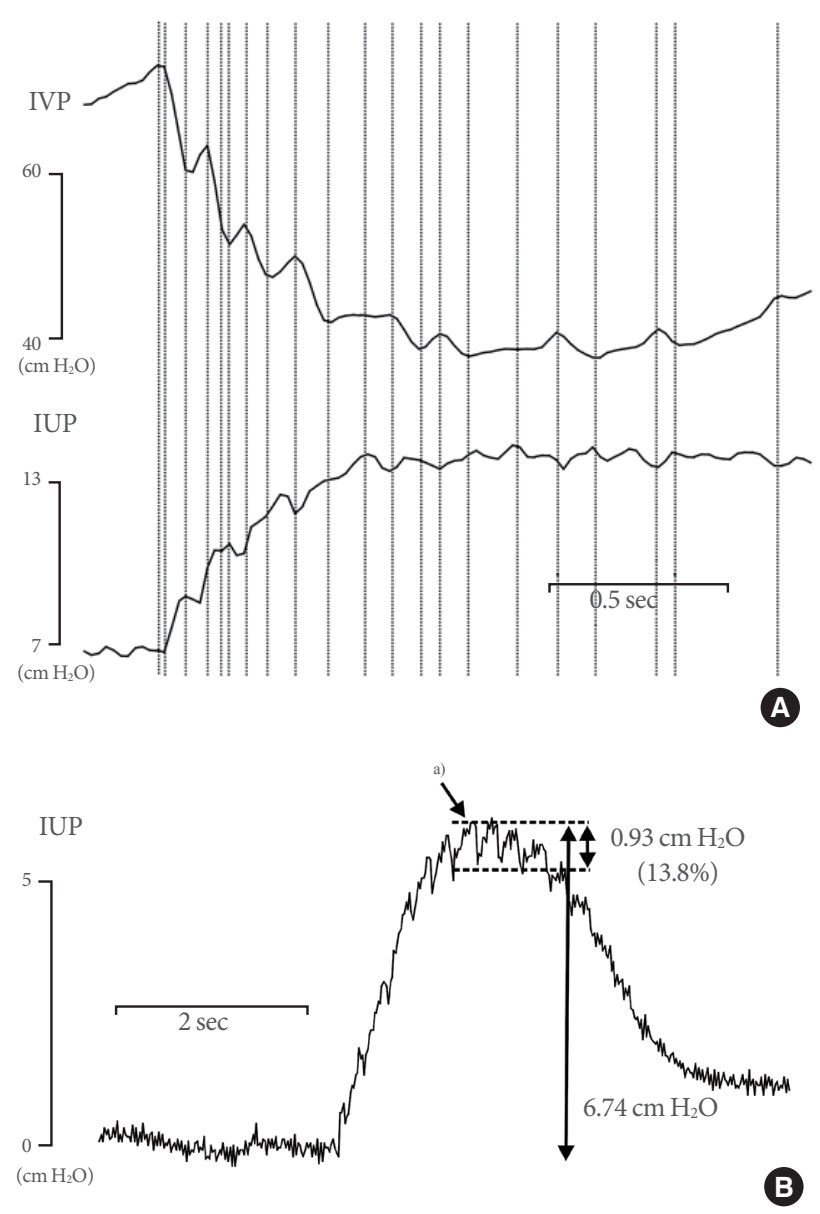

Fig. 4. Representative tracings showing intraluminal pressure high-frequency oscillations (IPHFOs). During IPHFOs, the imprints on the curves of intravesical pressure (IVP) and intraurethral pressure (IUP) exhibited an inverse relationship (A). The amplitude of IUP changes during IPHFOs corresponded to $13.8 \%$ of the maximal IUP increase (B). ${ }^{\text {a }}$ IUP changes during IPFHOs.

\section{Statistical Analysis}

The results are given as mean values \pm standard errors of the mean. Normal distributions were confirmed using the ShapiroWilk test. Statistical significance was determined using unpaired or paired Student t-tests or a one-way analysis of variance using Tukey post hoc test for multiple comparisons. All analyses were performed with GraphPad Prism ver. 5.03 (GraphPad Software Inc., La Jolla, CA, USA). Statistical significance was considered at values of $\mathrm{P}<0.05$. First, we compared the cystometric pressure and volume-related parameters of groups I and II to those of group III (controls) to investigate the effect of the additional catheters on the IVP catheter during voiding. Second, all rats in groups I and II were used to correlate by time the various voiding events on IVP, such as FSP or MP, to those on IUP, such as IUR or IUF, on their simultaneous curves. Third, the rats in group II were used to assess the FSP according to specific shapes on the DP curve, which was subtracted from IVP by IAP (Fig. $4 \mathrm{~A})$. And finally, we observed which correlation between the curve shapes of IVP and IUP during IPHFOs.

\section{RESULTS}

\section{Effects of Inserted Intraurethral Catheters and/or Intra- abdominal Balloons in Addition to Intravesical Catheters on Urodynamic Parameters}

The micturition patterns of rats from all groups were generally consistent and similar detailed features were seen in all cycles. Baseline urodynamic results from the three groups are shown in Table 1. There were no differences in any of the parameters of pressure and volume among the three groups, with the exception of increased MP in rats with the IUP catheter. Moreover, rats with the IUP catheter had significantly higher mortality rates than those without it.

Table 1. Comparison of cystometric parameters in conscious rats with catheters in the bladder, urethra, and/or abdomen

\begin{tabular}{lcccccccc}
\hline & $\mathrm{BP}\left(\mathrm{cm} \mathrm{H}_{2} \mathrm{O}\right)$ & $\mathrm{FSP}\left(\mathrm{cm} \mathrm{H}_{2} \mathrm{O}\right)$ & $\mathrm{TP}\left(\mathrm{cm} \mathrm{H}_{2} \mathrm{O}\right)$ & $\mathrm{MP}\left(\mathrm{cm} \mathrm{H}_{2} \mathrm{O}\right)$ & $\mathrm{BC}(\mathrm{mL})$ & $\mathrm{MV}(\mathrm{mL})$ & $\mathrm{RV}(\mathrm{mL})$ & $\mathrm{MI}(\mathrm{min})$ \\
\hline Group I & $16.1 \pm 4.7$ & $42.8 \pm 6.3$ & $38.2 \pm 7.4$ & $89.7 \pm 12.8$ & $1.2 \pm 0.7$ & $1.2 \pm 0.7$ & $0.0 \pm 0.0$ & $3.8 \pm 2.4$ \\
Group II & $16.8 \pm 1.9$ & $37.7 \pm 5.0$ & $32.6 \pm 3.1$ & $85.0 \pm 6.1$ & $1.1 \pm 0.4$ & $1.1 \pm 0.4$ & $0.0 \pm 0.0$ & $3.6 \pm 1.2$ \\
Group III & $10.5 \pm 1.2$ & - & $25.9 \pm 2.1$ & $65.8 \pm 6.1^{*}$ & $1.6 \pm 0.4$ & $1.5 \pm 0.4$ & $0.1 \pm 0.1$ & $4.6 \pm 1.2$ \\
\hline
\end{tabular}

Values are presented as mean \pm standard error of the mean.

$\mathrm{BP}$, basal pressure; FSP, flow starting pressure; TP, threshold pressure; MP, micturition pressure; BC, bladder capacity; MV, micturition volume; RV, residual volume; MI, micturition interval.

Group I: rats with two catheters in the bladder and urethra; group II: rats with three catheters in the bladder, perivesical space, and urethra; group III: rats with only one catheter in the bladder.

Comparisons were made among the three groups, but there were no significant differences except one (unpaired Student t-test).

${ }^{*} \mathrm{P}<0.05$ vs. group I. 


\section{FSP on IVP Curve Determined by IUP}

To investigate which point on the IVP or volume curve corresponded to that of IUP change, we checked and compared the time differences from the same TmP1 to points on the simultaneous curves of IVP, IUP, and volume (Figs. 2B, 3A, 3B). To determine FSP on the IVP curve, we compared the time differences from TmP1 to TmP2 on the IVP curve with IUR on the IUP curve. We also checked and compared these values to the VR on the volume curve (Figs. 2B, 3A). Then, to observe whether the MP was checked between the IUR and IUF, representing the urethral flow, we compared the time differences from TmP1 to TmP4, IUR, and IUF (Figs. 2B, 3B). Three reproducible micturition cycles from each rat of groups I and II were selected, and a total of 36 micturition cycles were used to find the FSP on IVP by IUP. The time difference from TmP1 to TmP2 $(2.31 \pm 0.44$ seconds) was not significantly different than the time from TmP1 to IUR ( $2.30 \pm 0.44$ seconds), which suggested that FSP corresponded to TmP2 on the IVP curve (Fig. 3C). The time difference from TmP1 to VR ( $4.92 \pm 0.30$ seconds) was significantly longer than that from TmP1 to IUR ( $3.09 \pm 0.31$ seconds), which suggested that volume tracing in awake cystometry was delayed by 1.83 seconds from the start of urethral leaking. This time delay seemed to be attributed to the time it took to pass through the collecting part of the metabolic cage below the rat. The FSP was $42.8 \pm 6.3 \mathrm{~cm} \mathrm{H}_{2} \mathrm{O}$, which was slightly higher than $\mathrm{TP}\left(38.2 \pm 7.4 \mathrm{~cm} \mathrm{H}_{2} \mathrm{O}\right)$, although this difference was not significant (Table 1).

\section{IPHFOs}

During the second phase, between $\mathrm{TmP} 2$ and $\mathrm{TmP} 3$, we found high-frequency oscillatory imprints on the IVP and IUP curves (Fig. 4). During the moments of oscillation, a rapid small increase in IVP was followed by a rapid small decrease, which was continuously repeated. The imprints on the IVP and IUP curves showed an inverse relationship between the oscillations. Although the imprint on IVP ascended, the simultaneous imprint on IUP descended, while that on IUP ascended at the moment of the IVP decrease (Fig. 4A). During the urethral flow, the IUP did not decrease to 0 , even during the contractile moments of the IPHFOs, and the amplitude of the IUP changes during IPHFO corresponded to $<15 \%$ of the maximal IUP increase (Fig. 4B).

\section{Micturition Pressure}

Among the 36 voiding cycles, 34 (94.4\%) exhibited a longer time difference from $\mathrm{TmP} 1$ to $\mathrm{TmP} 4$ than from TmP1 to IUF, while two (5.6\%) showed no difference. The time difference from $\mathrm{TmP} 1$ to $\mathrm{TmP} 4(8.04 \pm 0.29$ seconds) was significantly longer than that from TmP1 to IUF ( $7.14 \pm 0.30$ seconds) (Fig. $3 \mathrm{C})$. This finding suggests that MP does not occur during the simultaneous urethral flow checked by IUP.

\section{FSP on IVP Curve Determined by DP Curve and Comparison to Those by IUP}

The FSP on the IVP curve of awake animals is usually determined by the IAP, which makes it difficult to find the FSP using the IVP curve. After removal of the effect of IAP from the IVP curve, the DP curve clearly exhibited the shape of a kick-off point (KOP) around $\mathrm{TmP} 2$ (Fig. $5 \mathrm{~A}$ ). Using $\mathrm{TmP} 1$ as a reference point, we measured and compared the time differences to the KOP on the DP curve and the IUR on the simultaneous DP and IUP curves during the voiding phase. The FSP was $37.7 \pm 5.0 \mathrm{~cm}$ $\mathrm{H}_{2} \mathrm{O}$, which was slightly higher than $\mathrm{TP}\left(32.6 \pm 3.1 \mathrm{~cm} \mathrm{H}_{2} \mathrm{O}\right)$, although this difference was not significant (Table 1).

Fig. 5 shows an example of the cystometry, for which it was impossible to identify the FSP using only the IVP curve shape. After removing the superimposed effects of IAP from IVP, the DP curve clearly showed the shape of a KOP, which was located between $\mathrm{TmP1}$ and $\mathrm{TmP} 3$ (Fig. 5A). The time difference from TmP1 to KOP ( $4.44 \pm 0.35$ seconds) on the DP curve did not differ significantly from that of TmP1 to IUR $(4.44 \pm 0.36 \mathrm{sec}-$ onds), which suggested that the FSP could be clearly identified from the DP curve at TmP2 with a KOP appearance (Fig. 5B). Therefore, these results suggested the possibility of using the IAP in place of the IUP to detect the FSP.

\section{DISCUSSION}

Using our animal models with simultaneous registrations of IVP and IUP for awake cystometry, we demonstrated that urinary flow starts at the initial point of the second phase of voiding in IVP curves, similar to the previously reported anesthetic cystometry results. Furthermore, the FSP can easily be identified in the DP curve of awake cystometry from IVP and IAP without problematic IUP, which was shown by comparing the results measured with the IUP curve. These IAP measurements can be made without any injury to the rat as previous articles have shown [9,12-17], and they may be used as another important tool in voiding dysfunction research. According to the IUP curve, the conventionally known MP is checked after the ure- 

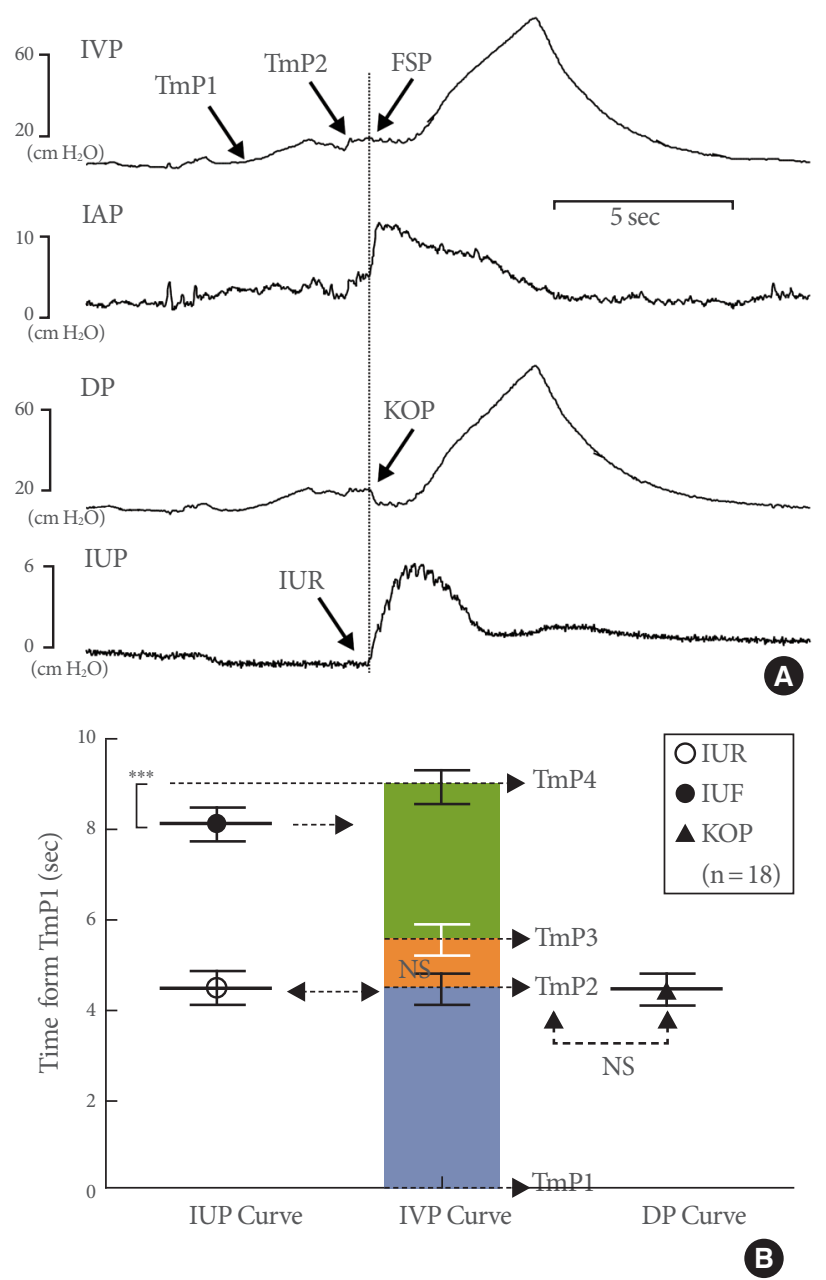

Fig. 5. Time differences from time point $1(\mathrm{TmP} 1)$ to various events on the simultaneous intravesical pressure (IVP), intraurethral pressure (IUP), and detrusor pressure (DP) curves from intra-abdominal pressure (IAP). (A) Representative tracings showing the simultaneous curves during the voiding phase. (B) Comparison of data from the curves of IUP, IVP, and volume (VOL) (mean \pm standard error). In some cystometric results, TmP2 could not be identified using only IVP shape. The IUP rise (IUR) on the IUP curve corresponds to the kick-off point (KOP) on the DP curve. NS, not significant. ${ }^{* * *} \mathrm{P}<0.001$ (compared to the IVP curve data).

thral flow ends. Thus, this pressure may be better termed "maximal micturition-related pressure" rather than MP because it does not occur during urethral flow.

Pressure is defined as a force from media perpendicular to the unit surface area [18]. Although this usually represents a scalar quantity, the IUP in this study was used as a tool to show the urethral flow start time as well as the IVP pressure at this moment, known as FSP on the curve of simultaneous IVP and
IUP registrations. During the filling phase, urethral pressure by IUP was at 0 because the tip of the urethral catheter in the distal urethra was buried in the coapted urethral mucosal walls. Just prior to voiding, the IUP became slightly negative, which is suspected to be due to the initiation of active urethral relaxation without urethral flow. As voiding started, the effect of urethral flow through the cracking urethra was instantly delivered to the urethral catheter tip that was used as the landmark for the voiding start. Using this IUP, we proved that the FSP also corresponds to the initial point of the second phase during awake rat voiding. This finding is comparable to study results of anesthetized rat voiding $[4,6,10]$. This point at which the flow increases in the volume curve does not represent the real flow start because of the delay caused by urine collection in the metabolic cage. Although the difference was not significant, there was a trend of a longer delay with less voided volume.

Micturition starts at the moment of urethral opening, which is caused by active urethral relaxation; this occurs just after detrusor contraction has been initiated [6,19-22]. This finding suggests that the FSP depends on the influence of initial urethral resistance rather than the maximal detrusor contraction power. In the clinical field, this pressure is similar to detrusor leak point pressure in patients with meningomyelocele, a pressure that is defined as the lowest DP at which urine leakage occurs [23]. An elevated detrusor leak point pressure $>40 \mathrm{~cm}$ $\mathrm{H}_{2} \mathrm{O}$, suggestive of increased urethral resistance, has been demonstrated as a risk factor for upper urinary tract deterioration in patients with myelomeningocele $[11,23,24]$. Therefore, this FSP, which has not been applied to animal models of disease such as bladder outlet obstruction, interstitial cystitis, or spinal cord injury may have some prognostic implications on the underlying mechanisms in the voiding dysfunction of these diseases.

The IPHFO is a phenomenon that results in oscillatory waves on the IVP curves during urinary flow and stems from the vibratory activities of the urethral striated rhabdosphincter, although controversy persists about the directions of wave progression. Based on the fact that, without the IPHFOs, the bladder contains significant amounts of residual urine [10,19,25,26], IPHFOs are presumed to be responsible for efficient voiding in male [6,25] and female [7] rats. However, their exact role in voiding remains controversial. One author suggested that the IPHFOs in the anesthetic animal model interrupt the urethral flow with on-off activity [6], which seems to be an opposing concept to efficient voiding. According to our results in awake animals, the IUP did not decrease to 0 even during the contrac- 
tile moments of IPHFOs, which was different than the results seen under anesthetized conditions. In addition, the amplitude of IUP changes during IPHFO corresponded to $<15 \%$ of the maximal IUP increase. These findings suggest that urethral flow is ongoing and not obstructed during IPHFOs, which seem to have a role in controlling the diameter of urethral flow according to pressure. During voiding, urine under high DP in a wider bladder may go through the narrow urethral wall, which gives rise to higher lateral pressure tension to the urethral wall. To resist this tension and maintain the appropriate urethral flow, the urethral wall must undergo appropriate tension changes from coaptation to a larger lumen diameter, which ideally needs to be vibratory for momentary accommodation. Thus, they show an inverse relationship between the simultaneous shapes of IPHFOs on the IVP and IUP curves during urethral flow [24]. They are known to stem from the contraction of the proximal urethra between the bladder and distal urethra [25].

The IUP decreased to almost 0 just after micturition, which was used as a landmark for the end of micturition. According to our result, $80 \%$ of MPs in the investigated voiding cycles were checked after the IUP decreased to 0 , which suggests that the MP was checked after urethral flow had ceased. One possible mechanism for this phenomenon would be the discordant movement between the movable bladder dome and the unmovable transvesical catheter grasped by fixation in the neck during voiding. In a human voiding cystogram, the full bladder, which expands to the level of the symphysis pubis in the lower abdomen, shrinks to the bladder neck with active voiding, which suggests that the dome in the proximal portion moves downward with active voiding. Because the catheter tip in the bladder dome does not move down to the bladder neck due to the solid character of the catheter, the pressure increases further by detrusor contraction without length change between them, similar to the mechanisms of muscle tension studies, during which the highest pressure is checked as MP.

While our IUP method is valuable for awake cystometry, there are certain limitations to its use. Although our technique is the most direct way to measure urethral pressure in the conscious rat, we cannot routinely use this method for rat disease model studies because of the associated morbidity and mortality. Furthermore, we could not apply this method in male rats because of the high mortality. Thus, we need to establish an alternative and less invasive method to replace IUP. The normal rat is known to use abdominal straining during every voiding, which is recorded as any increase in IAP just before voiding
$[9,27]$. This IAP increase added to IVP makes it difficult to discriminate the FSP shape on the IVP curve. Our results showed that the DP curve exhibits the peculiar shape of the FSP after removal of the effect of IAP from the IVP curve. This suggests that IAP allows for easier measurement of the FSP than IUP, which can be another advantage of IAP use in rat cystometry.

Rat cystometry is an essential tool for the indirect evaluation of human lower urinary tract functions and may be classified into anesthetic and awake types. Our awake IUP findings provide some similar and some differing information compared to findings from anesthetized rats. Despite their differences, such as the presence of consciousness, they provide complementary information on human voiding. Furthermore, our information, which could not be shown in anesthetic animals due to the differences in environment by the presence of consciousness, suggest aspects of human voiding that await further clarification.

\section{CONFLICT OF INTEREST}

No potential conflict of interest relevant to this article was reported.

\section{ACKNOWLEDGEMENTS}

This research was supported by the Basic Science Research Program through the National Research Foundation of Korea (NRF) and funded by the Ministry of Education, Science and Technology (KRF-2008-521-E00085) and the Korea Healthcare Technology R\&D Project, Ministry of Health \& Welfare, Republic of Korea Grant A090715-1002-0000100.

\section{REFERENCES}

1. Bridgewater M, MacNeil HF, Brading AF. Regulation of tone in pig urethral smooth muscle. J Urol 1993;150:223-8.

2. Bruschini H, Almeida FG, Srougi M. Upper and lower urinary tract evaluation of 104 patients with myelomeningocele without adequate urological management. World J Urol 2006;24:224-8.

3. Bennett BC, Kruse MN, Roppolo JR, Flood HD, Fraser M, de Groat WC. Neural control of urethral outlet activity in vivo: role of nitric oxide. J Urol 1995;153:2004-9.

4. Lee T, Andersson KE, Streng T, Hedlund P. Simultaneous registration of intraabdominal and intravesical pressures during cystometry in conscious rats--effects of bladder outlet obstruction and intravesical PGE2. Neurourol Urodyn 2008;27:88-95. 
5. Lee T, Yoon SM. The role of intra-abdominal pressure measurement in awake rat cystometry. Int Neurourol J 2013;17:44-7.

6. Fowler CJ, Griffiths D, de Groat WC. The neural control of micturition. Nat Rev Neurosci 2008;9:453-66.

7. Jin LH, Andersson KE, Han JU, Kwon YH, Park CS, Shin HY, et al. Persistent detrusor overactivity in rats after relief of partial urethral obstruction. Am J Physiol Regul Integr Comp Physiol 2011;301: R896-904.

8. Jin LH, Shin HY, Yoon SH, Seong DH, Park CS, Lee T, et al. Timerelated changes in detrusor overactivity in awake rats with spinal cord injury observed by simultaneous registrations of intravesical and intraabdominal pressures. Int Neurourol J 2011;15:19-24.

9. Jin LH, Lee HJ, Shin HY, Choi BH, Yoon SM, Park CS, et al. Development and changes with age of detrusor overactivity in spontaneous hypertensive rats as observed by simultaneous registrations of intravesical and intraabdominal pressures. Int Neurourol J 2011;15: 192-8.

10. Jin LH, Park CS, Shin HY, Yoon SM, Lee T. Dissimilar effects of tolterodine on detrusor overactivity in awake rats with chemical cystitis and partial bladder outlet obstruction. Int Neurourol J 2011;15:120-6.

11. Russo A, Castiglione F, Salonia A, Benigni F, Rigatti P, Montorsi F, et al. Effects of the gonadotropin-releasing hormone antagonist ganirelix on normal micturition and prostaglandin E(2)-induced detrusor overactivity in conscious female rats. Eur Urol 2011;59: 868-74.

12. Andersson KE, Wein AJ. Pharmacology of the lower urinary tract: basis for current and future treatments of urinary incontinence. Pharmacol Rev 2004;56:581-631.

13. Maggi CA, Santicioli P, Meli A. The nonstop transvesical cystometrogram in urethane-anesthetized rats: a simple procedure for quantitative studies on the various phases of urinary bladder voiding cycle. J Pharmacol Methods 1986;15:157-67.

14. McGuire EJ, Woodside JR, Borden TA, Weiss RM. Prognostic value of urodynamic testing in myelodysplastic patients. J Urol 1981;126:205-9.

15. Yoshimura N, Chancellor MB. Differential diagnosis and treatment of impaired bladder emptying. Rev Urol 2004;6 Suppl 1:S24-31.
16. Chien CT, Yu HJ, Lin TB, Chen CF. Neural mechanisms of impaired micturition reflex in rats with acute partial bladder outlet obstruction. Neuroscience 2000;96:221-30.

17. Cruz Y, Downie JW. Sexually dimorphic micturition in rats: relationship of perineal muscle activity to voiding pattern. Am J Physiol Regul Integr Comp Physiol 2005;289:R1307-18.

18. Merriam-Webster Dictionary. Pressure [Internet]. Springfield (MA): Merriam-Webster Inc.; c2013 [cited 2013 Nov 6]. Available from: http://www.merriam-webster.com/dictionary/pressure.

19. Conte B, Maggi CA, Parlani M, Giachetti A. Evidence for the existence of a urethro-urethral excitatory reflex in urethane anesthetized rats: involvement of peripheral ganglionic structures. J Urol 1991;146:1627-30.

20. Conte B, Maggi CA, Parlani M, Lopez G, Manzini S, Giachetti A. Simultaneous recording of vesical and urethral pressure in urethane-anesthetized rats: effect of neuromuscular blocking agents on the activity of the external urethral sphincter. J Pharmacol Methods 1991;26:161-71.

21. Kim KH, Jin LH, Choo GY, Lee HJ, Choi BH, Kwak J, et al. Nonselective blocking of the sympathetic nervous system decreases detrusor overactivity in spontaneously hypertensive rats. Int J Mol Sci 2012;13:5048-59.

22. Kruse MN, Belton AL, de Groat WC. Changes in bladder and external urethral sphincter function after spinal cord injury in the rat. Am J Physiol 1993;264(6 Pt 2):R1157-63.

23. Kontani H, Shiraoya C. Sex difference in urethral response to electrical stimulation of efferent nerves in the pudendal sensory branch of rats. Int J Urol 2002;9:586-95.

24. Streng T, Santti R, Talo A. Similarities and differences in female and male rat voiding. Neurourol Urodyn 2002;21:136-41.

25. Streng T, Santti R, Andersson KE, Talo A. The role of the rhabdosphincter in female rat voiding. BJU Int 2004;94:138-42.

26. Streng T, Santti R, Talo A. Possible action of the proximal rhabdosphincter muscle in micturition of the adult male rat. Neurourol Urodyn 2001;20:197-210.

27. Watanabe T, Constantinou CE. Analysis of pressure/flow characteristics in the female rat and their pharmacologic modulation. Neurourol Urodyn 1996;15:513-27. 